\title{
covid-19 Establishing a remote clinical advice service during the COVID-19 pandemic
}

\author{
Authors: Samuel Burrows, ${ }^{\mathrm{A}}$ Claire Asby, ${ }^{\mathrm{B}}$ Sarah E Ibitoye, ${ }^{\mathrm{C}}$ James Dodd, ${ }^{\mathrm{D}}$ Andrea Joughin, ${ }^{\mathrm{D}}$ Amy Crees, \\ Zoe Jones ${ }^{\mathrm{E}}$ and Katie Rainey ${ }^{\mathrm{F}}$
}

Service redevelopment has taken place across the NHS in response to the COVID-19 pandemic. At North Bristol NHS Trust, six vulnerable medical staff in non-patient facing roles set up a virtual advice service called 'Ask the Medical Reg'. This service aimed to provide senior medical support to inpatient and community teams for general medical and COVID-19related queries. Here we outline the structure of our service and present data from the first 4 weeks of operation. We describe how the service has supported both junior doctors working within the hospital and GPs and paramedics, helping with complex decisions to prevent unnecessary admissions.

KEYWORDS: coronavirus, remote consultation, delivery of healthcare, frailty, telemedicine

DOI: $10.7861 /$ fhj.2020-0092

\section{Problem}

Protecting vulnerable medical staff is one of many challenges for NHS hospitals during the COVID-19 pandemic. This resulted in the need to find new roles to accommodate staff unable to continue their usual patient-facing work due to their own personal health, as advised by NHS Employers.

Such workforce change came at a time when pressures on acute hospitals were predicted to rise, and pre-existing staff shortages were exacerbated by COVID-19-related illness. ${ }^{2,3}$ Loss of workplace identity and social isolation threatened the wellbeing of those affected, and trainees faced uncertainty about how this would affect their education and progression of training. ${ }^{4}$

\section{Potential solution}

Six vulnerable registrars and consultants in geriatric and acute medicine at North Bristol NHS Trust (NBT) established a virtual

Authors: Afoundation year 2 doctor, North Bristol NHS Trust, Bristol, UK; ${ }^{B}$ Clinical fellow, North Bristol NHS Trust, Bristol, UK; ${ }^{C}$ acute medicine registrar, North Bristol NHS Trust, Bristol, UK; ${ }^{D}$ care of the elderly registrar, North Bristol NHS Trust, Bristol, UK; Eacute medicine consultant, North Bristol NHS Trust, Bristol, UK; ' $c$ care of the elderly consultant, North Bristol NHS Trust, Bristol, UK advice service called 'Ask the Medical Reg' (ATMR). This service aimed to support patient-facing medical teams and provide a meaningful role for these clinicians during the pandemic.

The scope of the service included:

> provision of senior medical advice to NBT junior doctors and GPS

> supporting GPs and paramedics in decision-making when the benefits of hospital admission are uncertain

$>$ reviewing outstanding investigation results for discharged patients

> 'silver trauma' reviews: proactive medical reviews of all patients over the age of 65 years admitted to NBT with major trauma (as the geriatricians who usually provided this service were redeployed to medicine).

Four registrars and two consultants responded to telephone and email requests for medical advice. Consultants provided training and clinical supervision through case discussion, feedback and completion of workplace-based assessments. Two SHO-grade doctors were responsible for following up outstanding investigations and carrying out service data analysis to inform service development. The service was publicised on the local clinical commissioning group website and via email communication, promotional posters and junior doctor WhatsApp groups. Two outpatient clinic rooms provided an office base for the team. Trust laptops enabled remote access to the Trust clinical systems for out-of-hours work.

The service was accessible via telephone or email 24 hours a day, 7 days a week. Email requests for advice were responded to within 24 hours. All external calls requesting to speak to the medical registrar on-call or the 'geriatrician of the day' were directed to our service. A list of silver trauma patients was emailed daily by major trauma practitioners and the ATMR clinicians contacted the relevant inpatient teams to provide telephone advice.

Advice was documented on a standardised record and stored on the Trust computer system. Twice-weekly team meetings facilitated discussion of complex cases and of operational challenges and potential solutions, as well as providing team updates on the rapidly changing inpatient and outpatient services and sharing service data to support service development.

\section{Data collection}

Data were collected prospectively, with the exception of data on frailty, which were assessed retrospectively by reviewing the record of advice documentation for the presence of frailty as an explicitly stated issue or a Clinical Frailty Scale score of 5 or 


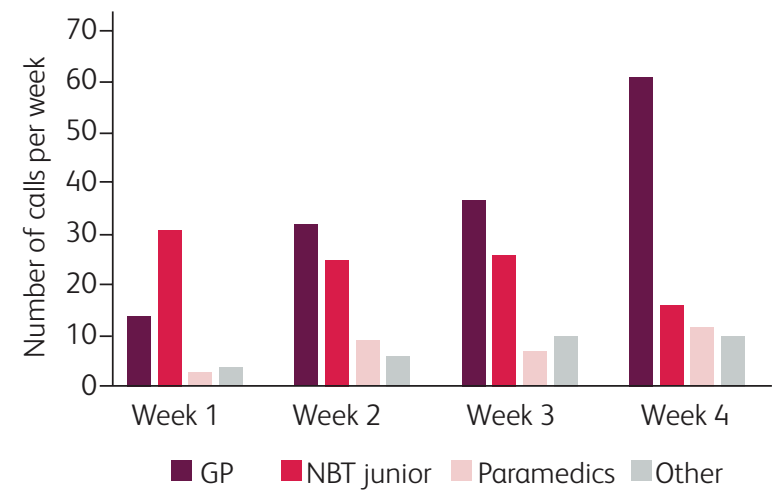

b

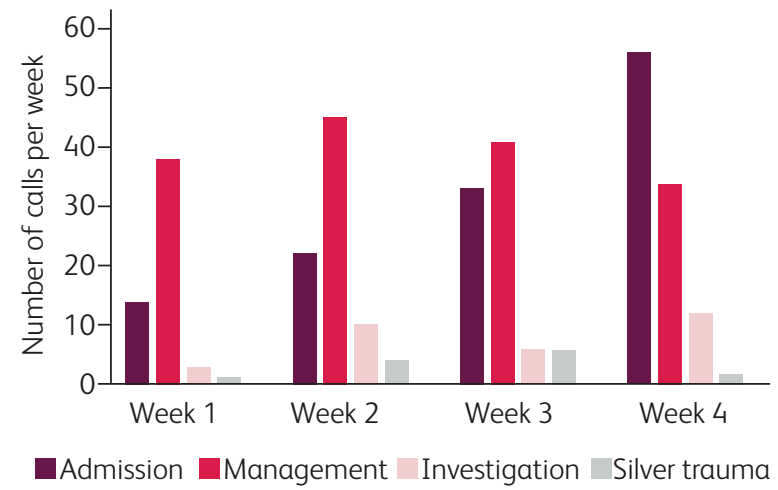

Fig 1. Calls to the 'Ask the Medical Reg' advice line per week categorised by a) referrer and b) theme. NBT = North Bristol NHS Trust.

more. ${ }^{6}$ If these were not documented, a geriatrician reviewed the patient's electronic medical records to look for markers of frailty. Consultations were categorised into themes including admission decision, management, investigation, 'silver trauma' reviews and COVID-19-related queries. Outcomes were defined as decision to admit, admission avoided, face-to-face review recommended, treatment advice, or specialist referral.

A questionnaire to evaluate service user experience was sent via email at the end of week 4 . Service utility was assessed using a Likert scale ( $1=$ not useful at all; $5=$ very useful) and whether the clinician would use the service again. Free text boxes were available for comments.

\section{Results}

\section{Referrals for clinical advice}

During the first 4 weeks of operation, the service received 303 requests for advice. Growth across this period was substantial, with a doubling of the workload from week 1 (mean [standard deviation; SD] calls per day: 7.43 [3.31]) to week 4 (mean [SD] calls per day:14.14 [5.70]). The median age was 71 years (range 19-100; interquartile range [IQR] 49.5-83); approximately a third $(38.6 \%)$ of patients were frail.

GPs were the most frequent service users, representing $47.5 \%$ of al requests for clinical advice. Other service users included NBT junior doctors (32.3\%) and paramedics (10.2\%). The proportions changed over the 4 weeks, with a falling number of calls for advice from NBT juniors and an increase in calls from GPs from week 1 to week 4 (Fig 1a). This was likely a consequence of internal restructuring of hospital teams improving inter-specialty collaboration and increased publicity of the service within primary care.

\section{Consultation theme and outcome}

The majority of consultations were queries about management (52.1\%) or potential admission (41.3\%) (Fig 1b). A quarter (25.7\%) of consultations were related to COVID-19, the proportion of which reduced over the 4 weeks ( $38.5 \%$ vs $24.2 \%$ ) in line with a national reduction in new COVID-19 diagnoses. Where advice was sought regarding potential admission, 55 of 125 patients (44\%) were admitted to hospital.

\section{Service users' experience}

There were 49 respondents to the online survey: 17 NBT doctors, 16 paramedics and $13 \mathrm{GPs}$. The majority of respondents indicated that they found the service useful or very useful (95.7\%) and would use the service again (93.7\%). Free text comments were positive, with many requesting that the service continue beyond the pandemic.

\section{Service providers' experience}

The team gained valuable experience in providing remote-based advice and were exposed to a wide range of medical problems. Trainees gained the opportunity to participate in service development and quality improvement. Additionally, team members gained a sense of purpose and contribution to the NHS's response to the COVID-19 pandemic.

\section{Conclusion}

This remote advice service was well received by patient-facing staff and provided a valuable role for vulnerable clinicians unable to participate in frontline work during the pandemic. Other benefits included admission avoidance in over half of patients for whom advice regarding hospital admission was sought, reducing pressure on acute hospital services and reducing the risk of nosocomial infection. Finally, we have demonstrated enthusiasm for a similar service to continue in the post-pandemic era, as a mechanism for primary care to gain rapid advice from secondary care clinicians regarding complex medical decision-making.

\section{Acknowledgements}

The authors would like to thank North Bristol NHS Trust for their support of vulnerable healthcare workers.

\section{References}

1 NHS Employers. Supporting our most vulnerable people. NHS Employers, 2020. www.nhsemployers.org/covid19/health-safetyand-wellbeing/supporting-our-most-vulnerable-people [Accessed 27 May 2020]. 
2 Royal College of Physicians. Focus on physicians: 2018-19 census (UK consultants and higher specialty trainees). RCP, 2019 Available from www.rcplondon.ac.uk/projects/outputs/focusphysicians-2018-19-census-uk-consultants-and-higher-specialtytrainees [Accessed 27 May 2020].

3 Royal College of Physicians. COVID-19 and its impact on NHS workforce. RCP, 2020. www.rcplondon.ac.uk/news/covid-19-and-itsimpact-nhs-workforce [Accessed 27 May 2020].

4 Joint Royal Colleges of Physicians Training Board. COVID-19 and recognition of trainee progression in 2020. JRCPTB, 2020. www. jrcptb.org.uk/news/covid-19-and-recognition-trainee-progression2020 [Accessed 27 May 2020].

5 British Geriatrics Society. COVID-19: Managing the COVID-19 pandemic in care homes for older people. BGS, 2020. www.bgs.org. uk/resources/covid-19-managing-the-covid-19-pandemic-in-carehomes [Accessed 27 May 2020].

6 Rockwood K, Song X, Macknight C et al. A global clinical measure of fitness and frailty in elderly people. CMAJ 2005;173:489-95.

7 Department of Health and Social Care and Public Health England. Number of coronavirus (COVID-19) cases and risk in the UK. DOHSC and PHE, 2020. www.gov.uk/guidance/coronaviruscovid-19-information-for-the-public [Accessed 27 May 2020].

Address for correspondence: Dr Andrea Joughin, Department for Care of the Elderly, Gate 10, level 6, Brunel Building, Southmead Hospital, Southmead, Bristol BS10 5NB, UK. Email: Andrea.Joughin@nbt.nhs.uk 